\title{
Equation of state and sound velocity of hadronic gas with hard-core interaction
}

\author{
L.M. Satarov ${ }^{1,2}$, K.A. Bugaev ${ }^{1,3}$, and I.N. Mishustin ${ }^{1,2}$ \\ ${ }^{1}$ Frankfurt Institute for Advanced Studies, D-60438 Frankfurt am Main, Germany \\ ${ }^{2}$ National Research Center "Kurchatov Institute", 123182 Moscow, Russia \\ ${ }^{3}$ Bogolyubov Institute for Theoretical Physics, 03680 Kiev, Ukraine
}

\begin{abstract}
Thermodynamic properties of hot and dense hadronic systems with a hard-sphere interaction are calculated in the Boltzmann approximation. Two parametrizations of pressure as a function of density are considered: the first one, used in the excluded volume model and the second one, suggested earlier by Carnahan and Starling. The results are given for one-component systems containing only nucleons or pions, as well as for chemically equilibrated mixtures of pions, nucleons and delta resonances. It is shown that the Carnahan-Starling approach can be used in a much broader range of hadronic densities as compared to the excluded volume model. In this case superluminal sound velocities appear only at very high densities, in the region where the deconfinement effects should be already important.
\end{abstract}

PACS numbers: 21.65.Mn, 25.75.-q, 25.75.Dw 


\section{INTRODUCTION}

Properties of strongly interacting matter under extreme conditions attract a great interest of researches working in several fields including relativistic heavy-ion collisions, physics of compact stars and the early universe. In recent years a significant progress was achieved in lattice calculations of the equation of state $(\mathrm{EoS})$ at high temperatures and low baryon densities. However, the lattice approach cannot be used reliably at low temperatures and high baryon densities. The information on the EoS in this domain remains a subject of model calculations. It is obvious that realistic calculations of the EoS of dense hadronic systems should take into account a strong interaction between hadrons.

A hard-sphere interaction (HSI) is one of the most popular methods to implement short range repulsion effects for calculating thermodynamic properties of multiparticle systems. In this approach the particles of a sort $i$ are represented by hard spheres of the radius $R_{i}$. It is assumed that particle move freely unless the distance $r_{i j}$ between centers of any pair $i, j$ becomes equal to $R_{i}+R_{j}$. It is postulated that the potential energy of $i j$-interaction is infinite at smaller $r_{i j}$. Originally such an approximation has been suggested by Van-der-Waals [1] to describe properties of dense gases and liquids. Later on the HSI-based models were successfully used by many authors in condensed matter physics [2, 3]. A similar approach, the so-called excluded volume model (EVM), has been applied in [4-10] to describe the EoS of hot and dense hadronic matter. These studies revealed a very strong sensitivity of the EoS to parameters of the short-range repulsion between hadrons. In particular, it has been shown in [5, 6] that a reasonable phase diagram of strongly interacting matter can only be obtained after accounting for finite sizes of hadrons. Our present study is aimed at a more realistic description of the HSI effects in the hadronic EoS.

Unfortunately, the Van-der-Waals approach is essentially nonrelativistic. As a consequence, it can not be safely applied when the sound velocity of matter $c_{s}$ becomes comparable with the light velocity. It is well known that the EVM violates the casuality condition ${ }^{1}$ $c_{s}<1$ at high enough baryon densities [6]. Attempts to remove this drawback were made in Refs. [7, 11] (see also [12, 13]). Moreover, it will be shown below that the EVM becomes inaccurate at high densities when the total volume of constituents exceeds $10-20 \%$ of the

\footnotetext{
1 Units $\hbar=c=1$ are used throughout the paper.
} 
system volume. By comparing with the virial expansion [2, 14] one may conclude that this model overestimates the contribution of non-binary interactions to pressure.

On the other hand, numerical simulations of one-component liquids with HSI show [2] that the Carnahan-Starling approximation (CSA) of pressure [15] successfully works up to much higher densities then in the EVM. Below we use the CSA and EVM to calculate properties of thermodynamically equilibrated hadronic systems containing mesons and baryons. For simplicity, we include into consideration only the lightest nonstrange hadrons (pions and nucleons) as well as the lightest baryonic resonance $\Delta$ (with the mass $m_{\Delta}=1232 \mathrm{MeV}$ ) in the zero-width approximation ${ }^{2}$. An important feature of such systems is that partial numbers of different species $N_{i}$ where $i=\pi, N, \Delta \ldots$ are, in general, not conserved due to presence of inelastic processes and resonance decays. These numbers are not independent and should be determined from the conditions of chemical equilibrium [6]:

$$
\mu_{\pi}=0, \mu_{N}=\mu_{\Delta}=\ldots=\mu_{B}
$$

Here $\mu_{i}$ is the chemical potential of the $i$-th species and $\mu_{B}$ is the baryon chemical potential. At given total baryon number $B$, system volume $V$ and temperature $T$ one can determine $\mu_{B}$ from the relation $\sum_{i} N_{i} b_{i}=B$ where $b_{i}$ is the baryon charge of the $i$-th species.

Up to now the information about properties of multi-component systems with HSI is rather scarce [3]. In this paper we consider several representative cases: first, we study the $N+\Delta$ and $\pi+N+\Delta$ mixtures with equal sizes of all hadrons and second, the $\pi+N+\Delta$ system assuming that baryons have equal radii, $R_{\Delta}=R_{N}$, and pions are point-like, $R_{\pi}=0^{3}$. The main emphasis is given to calculating the sound velocity. According to our analysis, the CSA predicts a much softer EoS, with smaller $c_{s}$-values than in the EVM. Choosing reasonable values of hadronic radii, we show that acasual states in the CSA are shifted to baryon densities $n_{B} \gtrsim 1 \mathrm{fm}^{-3}$. It is expected that at such densities the deconfinement effects, in particular, the formation of a quark-gluon phase should be already important.

The paper is organized as follows: in Sec. II we consider one-component systems with hard sphere particles. First we introduce parametrizations of pressure in the EVM and CSA. Then we calculate properties of an ideal gas in the Boltzmann approximation. Analytic expressions

\footnotetext{
2 We also neglect the isospin and Coulomb effects as well as possible creation of baryon-antibaryon pairs.

3 Note that small [9] or even vanishing [10] pion radii are favored by recent fits of hadron multiplicities observed in central heavy-ion collisions at the AGS, SPS and RHIC bombarding energies.
} 
for shifts of thermodynamic functions due to HSI are obtained in Sec. IIC and IID. The EoS and sound velocities of nucleon and pion matter are analyzed in Sec. IID and IIE. In Sec. III we study properties of the $N+\Delta$ and $\pi+N+\Delta$ mixtures. The summary and outlook are given in Sec. IV. In the Appendix we derive a general formula for the sound velocity of a relativistic gas.

\section{ONE-COMPONENT HADRONIC SYSTEMS}

\section{A. Compressibility and virial expansion}

In this section we consider a monodisperse system containing only one sort of hardsphere particles with radius $R$. Below we disregard the effects of Fermi or Bose statistics i.e. all calculations are done in the classical (Boltzmann) approximation. In this case one can write the following expression for pressure as a function of temperature and particle density $n=N / V[2,3]$ :

$$
P=n T Z(n)=P_{\mathrm{id}} Z(n)
$$

Here $P_{\text {id }}$ is the ideal gas pressure and $Z$ is the "compressibility" factor, which depends only on the dimensionless "packing" fraction $\eta=n v$ where $v=4 \pi R^{3} / 3$ is the proper volume of a single particle. At small $\eta$ one can use a universal virial expansion [2]

$$
Z=1+4 \eta+10 \eta^{2}+\ldots
$$

This expansion is not applicable ${ }^{4}$ for $\eta$ exceeding about 0.5. Equation (3) may be applied to estimate the accuracy of EoS calculations for multiparticle systems with HSI.

Instead of (3), different analytical approximations for $Z$ are used by many authors. For example, the following Van-der-Waals-motivated parametrization is used in the EVM:

$$
Z_{\mathrm{EVM}}=\frac{1}{1-4 \eta}
$$

One can see that such an ansatz leads to inaccurate results at high enough $\eta$. Indeed, comparison of the r.h.s of (4), decomposed in powers of $\eta$, with Eq. (3) shows that only

\footnotetext{
4 The most dense state of the considered systems corresponds to the ordered (face-centered cubic) lattice with $\eta=\frac{\pi}{3 \sqrt{2}} \simeq 0.74$. Direct Monte-Carlo simulations show [3] that the liquid-solid phase transition in a one-component matter with HSI occurs in the interval $0.49<\eta<0.55$.
} 
first two terms of the virial expansion are correctly reproduced in the EVM. It is clear that densities $n>0.25 / v$ can not be reached in this model due to the divergence of pressure at $\eta=0.25$. As demonstrated in Ref. [6], the EVM leads to superluminal sound velocities already at $\eta \gtrsim 0.2$. This is a consequence of a too stiff density dependence of pressure assumed in this model.

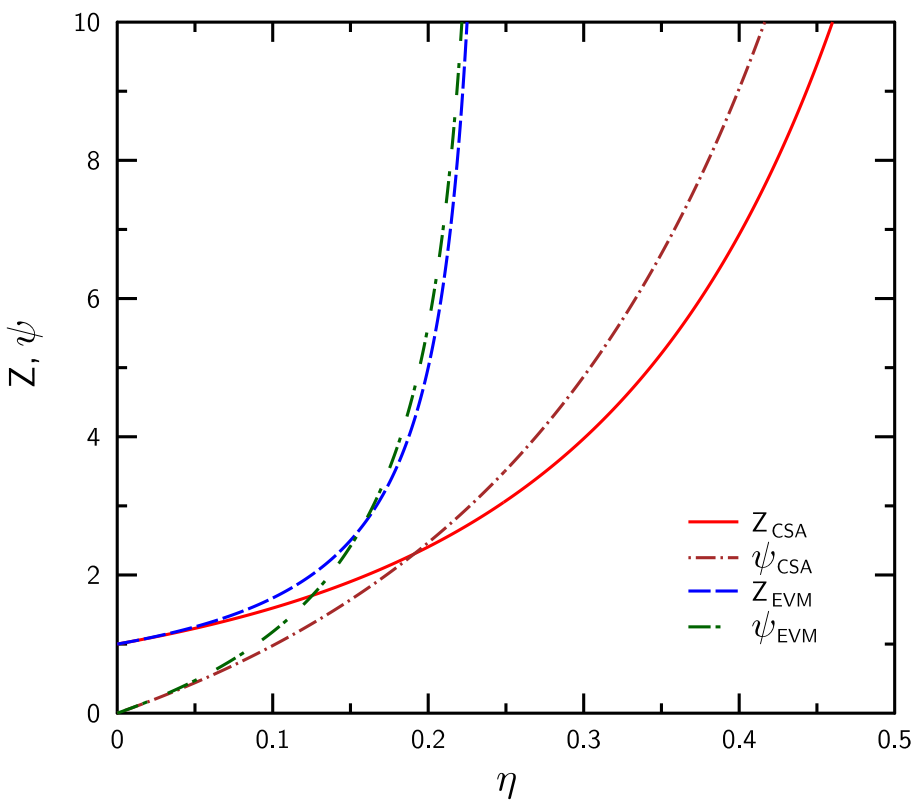

FIG. 1: (Color online) Compressibility factor $Z$ and function $\psi$ (see Eq. (22) ) for different values of packing fraction $\eta$ calculated within the EVM and CSA.

On the other hand, the Carnahan-Starling parametrization [15]:

$$
Z_{\mathrm{CSA}}=\frac{1+\eta+\eta^{2}-\eta^{3}}{(1-\eta)^{3}}
$$

is able to reproduce rather accurately [2] the first eight terms of the virial expansion for $Z(n)$. It agrees well with numerical calculations at $\eta \lesssim 0.5$ i.e. up to the boundary of liquid phase. Note that both above-mentioned parametrizations give similar results in the region $\eta \ll 1$ where $Z \simeq 1+4 \eta$. This is illustrated in Fig. 1, One can see that at $\eta \gtrsim 0.2$ the CarnahanStarling EoS is indeed noticeably softer as compared with the EVM.

Equation (2) gives pressure as a function of canonical variables: temperature $T$ and density $n$. As explained above, in the situation when particle densities are not fixed, more appropriate variables are temperature and chemical potential $\mu$. It is possible to calculate 
other thermodynamic functions, in particular the energy and entropy densities, $\varepsilon$ and $s$, if the dependence $\mu=\mu(T, n)$ is known. To get explicit expressions for these functions, it is convenient to calculate first the free energy density $f=\mu n-P$ as a function of $T$ and $n$. Then one can use thermodynamical identities [14]

$$
\varepsilon=f+T s, \quad s=-\left(\frac{\partial f}{\partial T}\right)_{n} .
$$

\section{B. Thermodynamic functions of ideal gas}

Let us start from calculating thermodynamic functions of an ideal gas of particles with the mass $m$ and the spin-isospin degeneracy factor $g$. In the Boltzmann approximation one can write down [6] the equation relating the particle density and the chemical potential

$$
n=\phi(T) \exp \left(\frac{\mu_{\mathrm{id}}}{T}\right), \phi(T) \equiv \frac{g m^{3}}{2 \pi^{2}} \frac{K_{2}(x)}{x} .
$$

Here $x=m / T, K_{n}(x)$ is the McDonald function of $n$-th order and the subscript 'id' implies the ideal gas limit. The function $\phi$ has the meaning of the ideal gas density in the case of zero chemical potential.

From Eq. (7) and formulae of preceding section we get the following expressions for thermodynamic functions of the ideal gas:

$$
\begin{aligned}
\mu_{\mathrm{id}} & =T \ln \frac{n}{\phi(T)}, \\
f_{\mathrm{id}} & =\mu_{\mathrm{id}} n-P_{\mathrm{id}}=n T\left[\ln \frac{n}{\phi(T)}-1\right], \\
s_{\mathrm{id}} & =n\left[\ln \frac{\phi(T)}{n}+\xi(T)\right] \\
\varepsilon_{\mathrm{id}} & =n T[\xi(T)-1]
\end{aligned}
$$

where

$$
\xi(T)=T \frac{\phi^{\prime}(T)}{\phi(T)}+1=x \frac{K_{3}(x)}{K_{2}(x)} .
$$

Unless otherwise stated, we denote by prime the derivative with respect to $T$. According to

Eq. (11), in the ideal gas limit, the heat capacity per particle $\widetilde{C}=n^{-1}\left(\frac{\partial \varepsilon}{\partial T}\right)_{n}$ is a function of temperature only:

$$
\widetilde{C}=[T(\xi-1)]^{\prime}=x^{2}+3 \xi-(\xi-1)^{2}
$$


The sound velocity is an important characteristics of EoS which gives the propagation speed of small density perturbations in the matter rest frame. In absence of dissipation the adiabatic sound velocity squared is equal to [16]

$$
c_{s}^{2}=\left(\frac{\partial P}{\partial \varepsilon}\right)_{\sigma},
$$

where the subscript $\sigma$ in the r.h.s. means that the derivative is taken along the Poisson adiabat, i.e. at constant entropy per particle ${ }^{5}: \sigma=s / n=$ const. One can rewrite Eq. (14) in the form

$$
c_{s}^{2}=\frac{(\partial P / \partial n)_{T}+(\partial P / \partial T)_{n}(\partial T / \partial n)_{\sigma}}{(\partial \varepsilon / \partial n)_{T}+(\partial \varepsilon / \partial T)_{n}(\partial T / \partial n)_{\sigma}} .
$$

Using Eqs. (10), (13) and the relation $d \sigma=d n \partial \sigma / \partial n+d T \partial \sigma / \partial T=0$ we get in the ideal gas limit

$$
n\left(\frac{\partial T}{\partial n}\right)_{\sigma}=\frac{T}{\widetilde{C}} .
$$

After calculating the derivatives of $P, \varepsilon$ in (15) and using Eq. (16) we obtain the following formula for the sound velocity of the monodisperse ideal gas:

$$
c_{s}^{\mathrm{id}}=\sqrt{\xi^{-1}\left(1+\widetilde{C}^{-1}\right)} .
$$

One can see that the sound velocity of the classical ideal gas is a function of temperature only.

In the nonrelativistic limit, $T \ll m$, using the asymptotic formulas for McDonald functions, one gets the approximate expressions

$$
\xi \simeq x+\frac{5}{2}+\frac{15}{8 x}+\ldots, \quad \widetilde{C} \simeq \frac{3}{2}+\frac{15}{4 x}-\frac{15}{2 x^{2}}+\ldots
$$

Substituting (18) into (17), we get the well-known non-relativistic expression $c_{s}^{\text {id }} \simeq \sqrt{\frac{5 T}{3 m}}$ for the sound velocity of a monoatomic ideal gas.

In the opposite, high temperature limit, $T \gg m$, one obtains from Eqs. (12)-(13)

$$
\xi \simeq 4+\frac{x^{2}}{2}+\ldots, \quad \widetilde{C} \simeq 3-\frac{x^{2}}{2}+\ldots
$$

This leads to the ultrarelativistic result $c_{s}^{\mathrm{id}} \simeq 1 / \sqrt{3} \simeq 0.577$. One can show that $c_{s}^{\mathrm{id}}(T)$ is a monotonically increasing function with the asymptotic value $1 / \sqrt{3}$.

\footnotetext{
5 In a general case, when particle numbers are not conserved $\sigma$ equals the entropy per baryon.
} 


\section{Contribution of HSI}

In this section we consider deviations from the ideal gas limit for particles with HSI. Let us denote by $\Delta A$ the shift of any quantity from its ideal gas value:

$$
\Delta A \equiv A-A_{\text {id }}
$$

It is clear that $\Delta A \rightarrow 0$ in the dilute gas limit $n \rightarrow 0$. Integrating the thermodynamic relation [14] $d \mu=\frac{1}{n}(d P-s d T$ ) along the density axis (at fixed $T$ ), one obtains the equation

$$
\Delta \mu(T, n)=\int_{0}^{n} \frac{d n_{1}}{n_{1}} \frac{\partial \Delta P\left(T, n_{1}\right)}{\partial n_{1}} .
$$

Here we have used the condition $\lim _{n \rightarrow 0} \Delta \mu=0$. Substituting $\Delta P=n T(Z-1)$, one arrives at the relation $\Delta \mu=T \psi(n)$ where

$$
\psi(n)=Z(n)-1+\int_{0}^{n} \frac{d n_{1}}{n_{1}}\left[Z\left(n_{1}\right)-1\right] .
$$

The same formula for $\Delta \mu$ has been obtained earlier in Ref. [17]. Using further Eq. (8) we finally get the equation for the chemical potential $\mu=\mu_{\text {id }}+\Delta \mu$ as a function of $T$ and $n$ :

$$
\mu=T\left[\ln \frac{n}{\phi(T)}+\psi(n)\right] .
$$

By solving (23) with respect to $n$ and substituting the result into Eq. (2) one can calculate pressure as a function of grand-canonical variables $T, \mu$. In particular, this may be useful for finding possible phase transitions by using the Gibbs construction. Parametrizations of the compressibility factor introduced in Sec. IIA are rather useful because they permit an analytical integration in Eq. (22). For example, in the EVM Eqs. (21), (41), (22) give the following result

$$
\psi_{\mathrm{EVM}}=Z-1+\ln Z=\frac{b P}{T}+\ln \left(1+\frac{b P}{T}\right)
$$

where $b=4 v$ is the "excluded volume" introduced by Van-der-Waals. Substituting (24) into (23) leads to a simple formula for baryon chemical potential

$$
\mu=T \ln \frac{P}{T \phi(T)}+b P \quad(\mathrm{EVM})
$$

One can regard Eq. (25) as the implicit equation for $P=P(T, \mu)$. In the considered case solving Eq. (25) with respect to $P$ is equivalent to solving Eq. (23) with respect to $n$. It is 
worth noting that in the EVM the shift of chemical potential from the ideal gas value $(b=0)$ is linear in pressure. But this conclusion is not universal: it does not hold in the CSA.

Indeed, substituting (51) into Eq. (22) gives the following formula

$$
\psi_{\mathrm{CSA}}=\frac{3-\eta}{(1-\eta)^{3}}-3 .
$$

In Fig. 1 we present numerical values of $\psi(n)$ in the EVM and CSA. One can see that at given $T, n$ the values of $\psi$ and, therefore, deviations of chemical potential from the ideal gas values are larger in the EVM.

\section{Nucleonic matter}

Let us consider first a system consisting of nucleons $(m=939 \mathrm{MeV}, g=4)$. In this case $n$ is the conserved baryon density, which together with temperature defines the thermodynamic state. At fixed $n$ and $T$ the shift of free energy density due to nucleon interactions equals $\Delta f=n \Delta \mu-\Delta P$. Using further Eqs. (9) and (22) we obtain the expression for the free energy density of interacting nucleons

$$
f=n T\left\{\ln \frac{n}{\phi(T)}-1+\int_{0}^{n} \frac{d n_{1}}{n_{1}}\left[Z\left(n_{1}\right)-1\right]\right\} .
$$

Equations (6), (27) lead to the following formulae for entropy and energy densities:

$$
\begin{aligned}
& s=n\left\{\ln \frac{\phi(T)}{n}+\xi(T)-\int_{0}^{n} \frac{d n_{1}}{n_{1}}\left[Z\left(n_{1}\right)-1\right]\right\}, \\
& \varepsilon=f+T s=n T[\xi(T)-1],
\end{aligned}
$$

where $\xi(T)$ is defined in Eq. (12).

From Eqs. (11) and (29) one can see that HSI does not produce any shift of the energy density as compared to the ideal gas of point-like nucleons ${ }^{6}$. As a consequence, the isochoric heat capacity $C_{\mathrm{V}}=(\partial \varepsilon / \partial T)_{n}$ is the same as in the ideal gas: $C_{\mathrm{V}}=n \widetilde{C}(T)$ where $\widetilde{C}(T)$ is given by Eq. (13). According to (28), the entropy per particle $\sigma=s / n$ is reduced due to hardcore interaction of nucleons. This leads to a modification of the Poisson adiabat ( $\sigma=$ const)

\footnotetext{
6 This result is rather obvious. It is clear that the energy per particle, $\varepsilon / n$, for one-component systems with classical hard-sphere particles should depend only on temperature, at least for densities below the liquid-solid transition. Therefore, increasing the density at fixed $T$ does not change $\varepsilon / n$.
} 


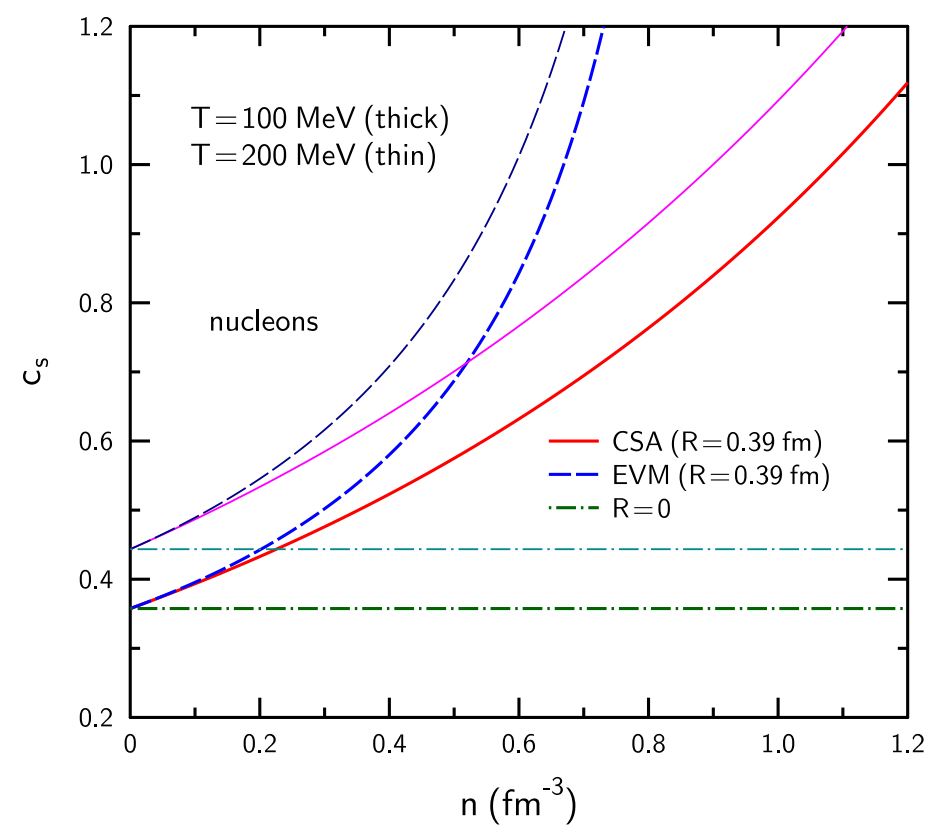

FIG. 2: (Color online) Sound velocity of nucleon gas as a function of density for two values of temperature $T=100$ (thick lines) and 200 (thin lines) MeV. The solid and dashed curves are calculated by using compressibility functions proposed in the CSA and EVM. The dash-dotted lines are obtained in the limit of point-like nucleons $(R=0)$.

in the $n-T$ plane as compared to the ideal gas. Indeed, using Eq. (28), we obtain for the isentropic process

$$
n\left(\frac{\partial T}{\partial n}\right)_{\sigma}=Z T \widetilde{C}^{-1} .
$$

Comparing this result with Eq. (16) we conclude that the slope of the Poisson adiabat of nucleonic matter increases with density due to the appearance of the compressibility factor $Z>1$.

The sound velocity can be obtained from Eqs. (22), (15), (29)-(30). This leads to the analytic expression

$$
c_{s}^{2}=\frac{1}{\xi+Z-1}\left[(n Z)^{\prime}+Z^{2} \widetilde{C}^{-1}\right],
$$

where prime means the derivative with respect to $n$. In the ideal gas limit $Z \rightarrow 1$ this formula coincides with Eq. (17). In the case of a nucleon gas at realistic temperatures $T \ll m$, using the relations (18) one can derive the approximate formula

$$
c_{s}^{2} \simeq \frac{(n Z)^{\prime}+2 Z^{2} / 3}{Z+x+3 / 2},
$$




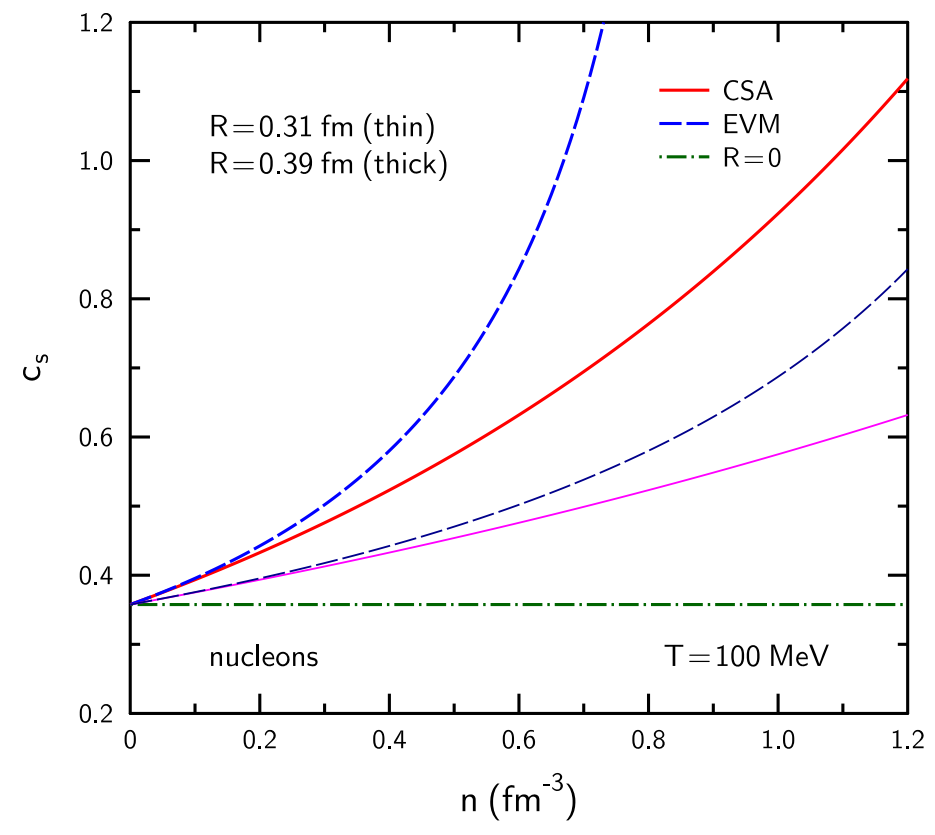

FIG. 3: (Color online) Sound velocity of nucleon matter as a function of density for different values of parameter $R$. The dashed and solid curves are calculated, respectively, in the EVM and CSA. The dash-dotted line corresponds to point-like nucleons.

where $x=m / T$. Equations (31)-(32) clearly show that HSI leads to superluminal sound velocities, $c_{s}>1$, at high enough densities where $Z$ is large (for further discussion, see [6]). This is especially evident in the EVM where $(n Z)^{\prime}=Z^{2}$. According to Eq. (32), in this case $c_{s}^{2}$ is proportional to $Z$ at large $Z$.

In Fig. 2 we compare the sound velocities values, calculated by using the parametrizations (44) and (5) for two typical values of temperature. We have chosen the nucleon radius $R=0.39 \mathrm{fm}$ which corresponds to the excluded volume $b=1 \mathrm{fm}^{3}$, used previously in Ref. [6]. Again one can see that the CSA predicts a much softer EoS (i.e. smaller $c_{s}$ ) than the EVM. Our calculations show, that at realistic temperatures $T \lesssim 200 \mathrm{MeV}$ the sound velocity in the CSA remains below unity up to rather large densities $n \simeq 0.9 \mathrm{fm}^{-3}$. On the other hand, superluminal sound velocities appear in the EVM at much smaller $n$. According to Fig. 2, deviations from the ideal gas limit $R \rightarrow 0$ become significant already at subnuclear densities $n \sim 0.1 \mathrm{fm}^{-3}$.

Figure 3 demonstrates that the sound velocity is very sensitive to the choice of the particle size $R$. Note that a $20 \%$ reduction of $R$, from 0.39 to $0.31 \mathrm{fm}$, corresponds to the two-fold 


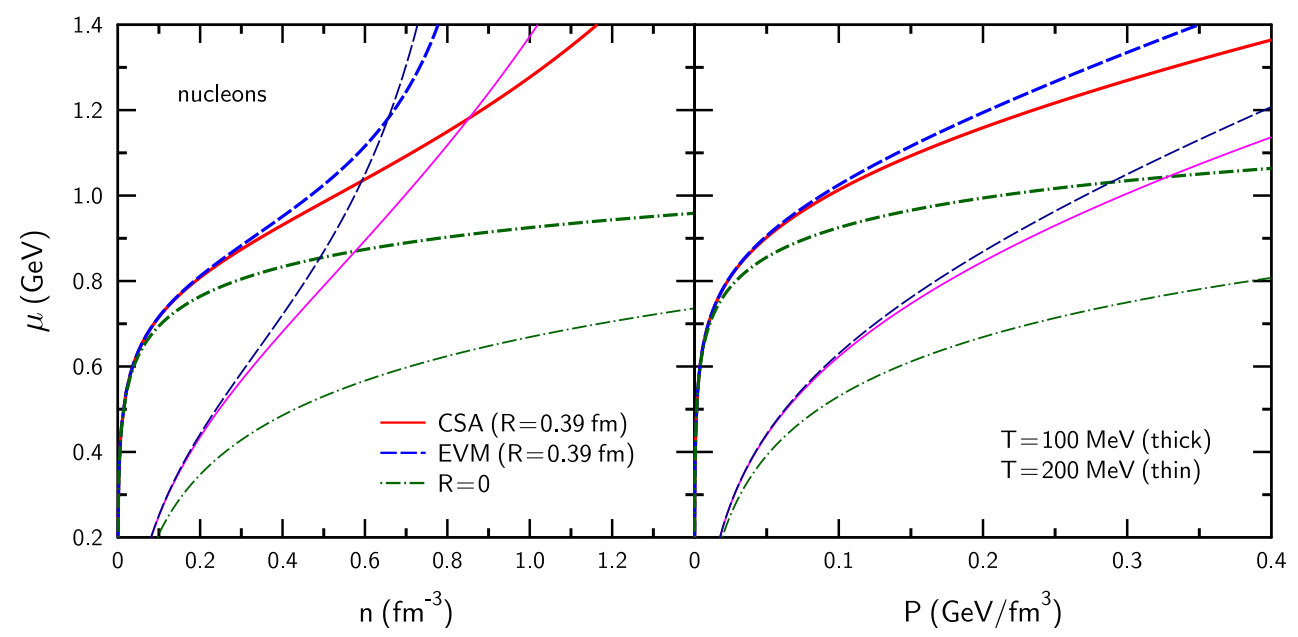

FIG. 4: (Color online) Chemical potential of nucleon gas as a function of density (left panel) and pressure (right panel) calculated in the CSA (solid lines) and EVM (dashed lines) at temperatures 100 and $200 \mathrm{MeV}$. The dashed-dotted lines correspond to point-like nucleons.

decrease of the excluded volume $b$. It is seen that the difference between CSA and EVM is smaller for lower $R$. Figure 4 shows the results for the baryon chemical potential as a function of nucleon density and pressure. One can see that at $n \gtrsim 0.4 \mathrm{fm}^{-3}$ the CSA indeed predicts significantly smaller values of $\mu$ as compared to the EVM. On the other hand, at given $\mu$ the pressure in the CSA is noticeably larger than in the EVM. This makes the nucleon phase more stable at high densities as compared to the EVM.

\section{E. Pion matter}

Let us consider now thermodynamic properties of matter composed of finite-size "thermal" pions with the vacuum mass $m_{\pi}=140 \mathrm{MeV}$ and the statistical weight $g_{\pi}=3$. As before, we assume the hard-sphere interaction of particles and perform all calculations in the Boltzmann approximation. To emphasize specific features of the pion system we introduce the subscript ' $\pi$ '. Using Eqs. (21), (23), one can write the following equations for pressure and chemical potential of pions

$$
P_{\pi}=T n_{\pi} Z\left(n_{\pi}\right), \quad \mu_{\pi}=T\left[\ln \frac{n_{\pi}}{\phi_{\pi}(T)}+\psi\left(n_{\pi}\right)\right],
$$

where $\phi_{\pi}$ and $\psi$ are defined in (7) and (22) (with $m=m_{\pi}, g=g_{\pi}$ ). 


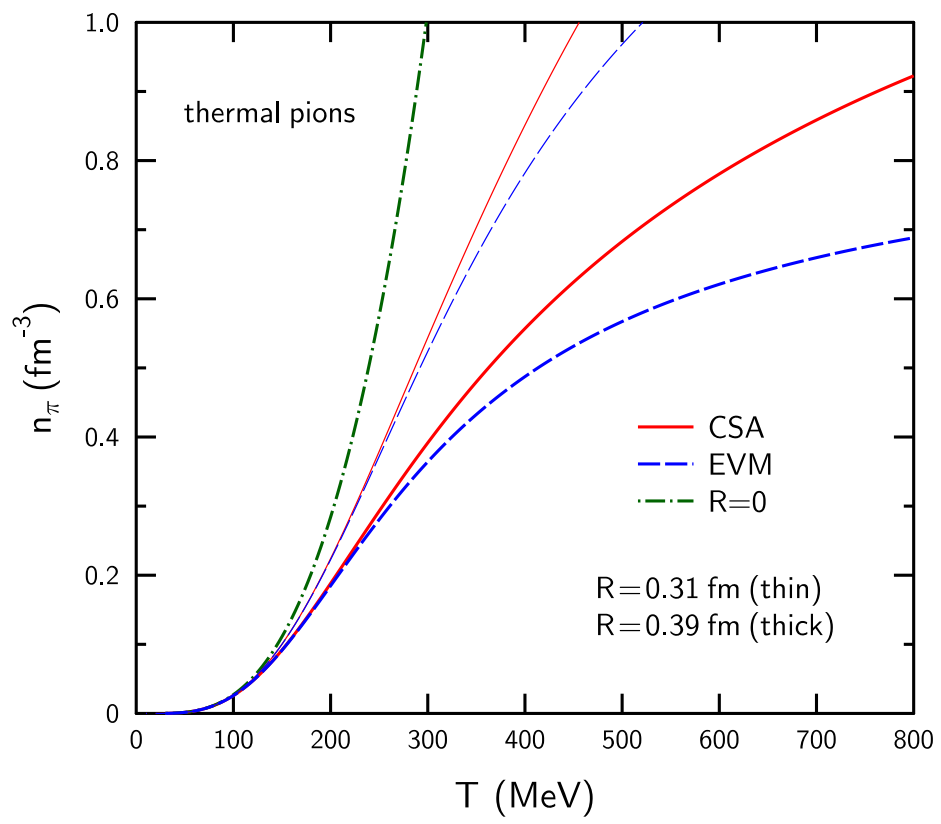

FIG. 5: (Color online) Equilibrium density of pions as a function of temperature for different values of parameter $R$. The dashed and solid curves are obtained, respectively, in the EVM and CSA. The dash-dotted line corresponds to ideal gas of point-like pions.

At fixed temperature one can find equilibrium values of density $n_{\pi}=n_{\pi}(T)$ and other thermodynamic functions from the condition of chemical equilibrium $\mu_{\pi}=0$. Then we obtain the following implicit equation for density of pions

$$
n_{\pi}=\phi_{\pi}(T) e^{-\psi\left(n_{\pi}\right)}
$$

As one can see from Eq. (34), finite size effects suppress the pion density as compared to the ideal gas limit $\psi \rightarrow 0$. This is illustrated in Fig. 5 where we compare the results of the EVM and CSA, for several values of the hadronic radius $R$. One can see noticeable deviations from the ideal gas already at $T \simeq 150 \mathrm{MeV}$, but a significant difference between the CSA and EVM calculations appears only at unrealistically high temperatures $T \gtrsim 400 \mathrm{MeV}$. Such a behavior follows from a relatively slow increase of pion packing ratio with temperature. Note that short-range repulsive interactions of pions should also suppress possible Boseenhancement effects at high temperatures.

One can easily calculate the entropy density of interacting pion gas. In the case $\mu_{\pi}=0$, 
using the thermodynamic relation $s_{\pi}=d P_{\pi} / d T$, one has

$$
s_{\pi}=T \frac{d n_{\pi}}{d T}\left(n_{\pi} Z\right)^{\prime}+n_{\pi} Z=n_{\pi}\left(Z+\xi_{\pi}-1\right)
$$

where prime denotes the derivative with respect to the density $n_{\pi}$ and $\xi_{\pi}$ is defined in Eq. (12). In the second equality we use the relation

$$
T \frac{d n_{\pi}}{d T}=\frac{n_{\pi}\left(\xi_{\pi}-1\right)}{1+n_{\pi} \psi^{\prime}}=\frac{n_{\pi}\left(\xi_{\pi}-1\right)}{\left(n_{\pi} Z\right)^{\prime}}
$$

which follows from Eq. (34) after taking the derivative with respect to $T$. According to Eqs. (35), (19) the entropy per pion $s_{\pi} / n_{\pi}$ equals approximately $Z+3$ at $T \gg m_{\pi}$. This value exceeds the corresponding ratio for massless point-like pions $(Z=1)$.

Equation (35) leads to the following formula for the energy density $\varepsilon_{\pi}=T s_{\pi}-P_{\pi}$ :

$$
\varepsilon_{\pi}=n_{\pi} T\left(\xi_{\pi}-1\right)
$$

From this result one can see that at given temperature the energy per particle is the same as in the ideal pion gas. Using (36)-(37), (13) we get the equation for the heat capacity per pion $\widetilde{C}_{\pi}=n_{\pi}^{-1} d \varepsilon_{\pi} / d T$ :

$$
\widetilde{C}_{\pi}=x_{\pi}^{2}+3 \xi_{\pi}+\left(\xi_{\pi}-1\right)^{2}\left[\frac{1}{\left(n_{\pi} Z\right)^{\prime}}-1\right]
$$

where $x_{\pi}=m_{\pi} / T$.

Finally we obtain the following formula for the sound velocity squared

$$
c_{s}^{2}=\frac{d P_{\pi}}{d \varepsilon_{\pi}}=\frac{s_{\pi}}{n_{\pi} \widetilde{C}_{\pi}}=\frac{Z+\xi_{\pi}-1}{\widetilde{C}_{\pi}} .
$$

In the ideal gas limit $Z \rightarrow 1$ one gets $c_{s}=\left(3+x_{\pi}^{2} / \xi_{\pi}\right)^{-1 / 2}=\left(3+x_{\pi} K_{2} / K_{3}\right)^{-1 / 2}$. By using (19) we arrive at the approximate relation

$$
c_{s}^{2} \simeq \frac{1}{3}\left(Z+n_{\pi} Z^{\prime}\right)\left(1+\frac{n_{\pi} Z^{\prime}}{Z+3}\right)^{-1},
$$

in the ultrarelativistic case $x_{\pi} \ll 1$. Figure 6 shows the results of $c_{s}$-calculations with the parameters $R=0.20$ and $0.39 \mathrm{fm}$. One can see that at $T \gtrsim 200 \mathrm{MeV}$ the obtained sound velocities noticeably exceed the asymptotic ideal gas value $c_{s}=1 / \sqrt{3}$. The calculations show, that these velocities become superluminal only at unrealistically high temperatures at which hadrons should melt [18, 19]. 


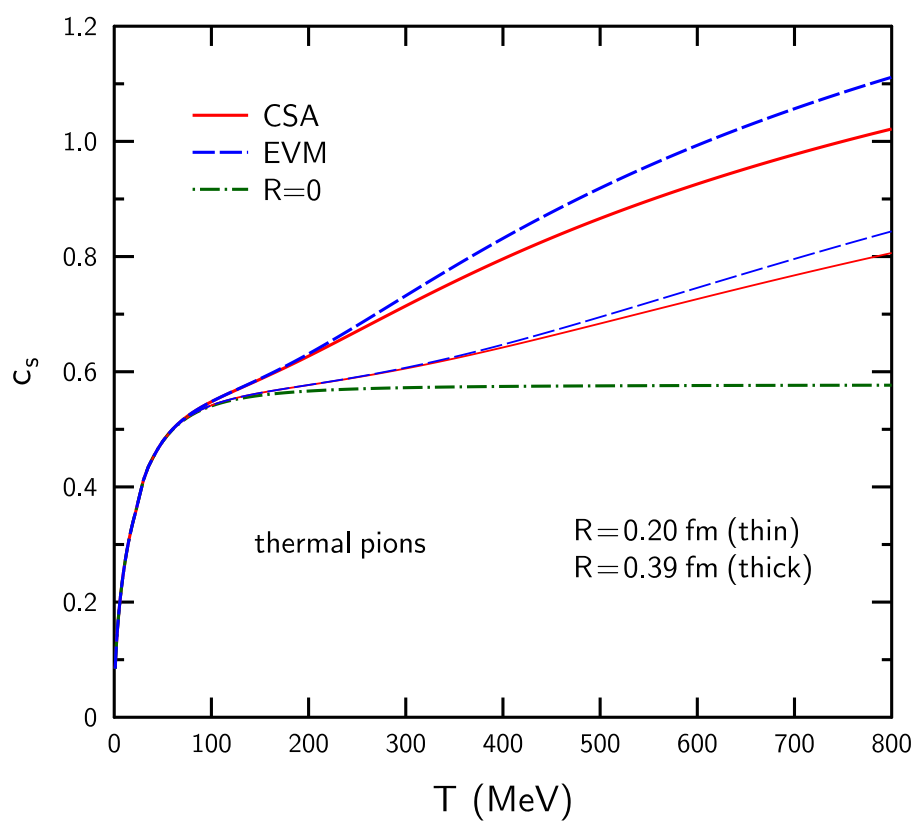

FIG. 6: (Color online) Sound velocity of pion gas as a function of temperature for different values of parameter $R$. The dashed and solid curves are calculated, respectively, in the EVM and CSA. The dash-dotted line corresponds to point-like pions.

\section{HADRONIC MIXTURES}

\section{A. General remarks}

Let us consider now a multi-component hadronic matter composed of particles of different kinds $i=1,2 \ldots$ Most detailed information about the EoS of this matter can be obtained if one knows its pressure $P=P\left(T, n_{1}, n_{2} \ldots\right)$ as a function of temperature $T$ and partial densities $n_{i}=N_{i} / V$. As before, we neglect the quantum effects and assume that particles interact via HSI. In this case one can write down [3] first two terms of the virial expansion of pressure in powers of $n_{i}$ :

$$
\frac{P}{n T}=1+\sum_{i, j} b_{i j} x_{i} x_{j}+\ldots
$$

Here $n=\sum_{i} n_{i}$ is the total density, $x_{i}=n_{i} / n$ and coefficients $b_{i j}=\frac{2 \pi n}{3}\left(R_{i}+R_{j}\right)^{3}$, where $R_{i}$ is the radius of the $i$-th species. If particle radii are the same $\left(R_{i}=R\right.$ for all $\left.i\right)$ the second term in the r.h.s. equals $4 \eta$ where $\eta=4 \pi R^{3} n / 3$. In this limit most of the results for oneand multi-component systems will be formally the same. In particular, one may use Eq. (2) 
and the formulae for thermodynamic functions from Sec. IID by identifying the variable $n$ with the total density of all species.

It is possible to calculate the shift of the free energy density, $\Delta f$, for any multi-component system if one knows its pressure as a function of temperature and partial densities. Below we use the method suggested in Ref. [22]. Using the thermodynamic relation $d F=-P d V$ for the change of total free energy $F$ in the isothermal process, one can write down the equation connecting the shifts of $F$ and $P$ :

$$
\Delta F=\int_{V}^{\infty} d V_{*} \Delta P\left(T, \frac{N_{1}}{V_{*}}, \frac{N_{2}}{V_{*}} \ldots\right) .
$$

The r.h.s. of this equation is equal to the work done by particle interactions during the isothermal compression of matter from an asymptotically large volume to $V_{*}=V$. Introducing the variable $\alpha=V / V_{*}$ one obtains the expression for $\Delta f=\Delta F / V$ :

$$
\Delta f=\int_{0}^{1} \frac{d \alpha}{\alpha^{2}} \Delta P\left(T, \alpha n_{1}, \alpha n_{2} \ldots\right) .
$$

For a one-component matter with HSI, substituting $\Delta P=n T(Z-1)$, we return to the formulae, obtained in Sec. 【ID.

It is easy to derive exact results for mixtures where one of the components consists of point-like particles. Namely, let us consider a two-component system where the ratio of particle radii $R_{2} / R_{1}$ is small. In the limit $R_{2} \rightarrow 0$ one can regard the component $i=2$ as an ideal gas but in the reduced "free" volume $\widetilde{V}=V-N_{1} v_{1}=V\left(1-\eta_{1}\right)$. Here $v_{1}$ and $\eta_{1}$ are, respectively, the proper volume and the packing fraction of particles $i=1$. The partial pressure of the first component may be written analogously to Eq. (22). This leads to the following equation for pressure of a two-component mixture with $R_{2} / R_{1} \ll 1[3]$ ]

$$
P\left(T, n_{1}, n_{2}\right)=n_{1} T Z\left(n_{1}\right)+\frac{n_{2} T}{1-\eta_{1}} .
$$

The last term is the partial pressure of the second component $P_{2}=\widetilde{n}_{2} T$. Here $\widetilde{n}_{2}=N_{2} / \widetilde{V}$ is the "local" density of particles $i=2$ which is larger than the "average" density $n_{2}=N_{2} / V$. Using Eq. (44) one can easily prove the validity of the virial theorem (41) in the limit $n_{1}, n_{2} \rightarrow 0$. In fact, instead of particles $i=1$ we can consider an arbitrary multi-component mixture composed of hadrons with the same radii. In this case $n_{1}$ equals the total density of such a mixture. 


\section{B. The $N+\Delta$ matter}

In this section we consider the EoS of a chemically equilibrated binary mixture of baryons: nucleons $(N)$ and the lightest $\Delta$ resonances $\left(m_{\Delta}=1232 \mathrm{MeV}, g_{\Delta}=16\right)$. This system is characterized by two canonical variables: temperature and the baryon density $n_{B}=n_{N}+n_{\Delta}$. We assume that all baryons have the same radii, i.e. $R_{N}=R_{\Delta}=R$. In this case HSI does not distinguish $N$ and $\Delta$, therefore, the hadronic pressure can be written as $P=n_{B} T Z\left(n_{B}\right)$. Same arguments as used in deriving Eq. (23) lead to the equation for chemical potential of the $i$-th species $(i=N, \Delta)$ :

$$
\mu_{i}=T\left[\ln \frac{n_{i}}{\phi_{i}(T)}+\psi\left(n_{B}\right)\right] .
$$

Here $\phi_{i}$ is defined in Eq. (77) with the replacement $m \rightarrow m_{i}, g \rightarrow g_{i}$.

From the condition of chemical equilibrium $\mu_{N}=\mu_{\Delta}=\mu_{B}$ we get the expressions

$$
\begin{aligned}
& \mu_{B}=T\left[\ln \frac{n_{B}}{\phi_{N}+\phi_{\Delta}}+\psi\left(n_{B}\right)\right], \\
& n_{i}=n_{B} w_{i}(T), \quad w_{\Delta}=1-w_{N}=\frac{\phi_{\Delta}}{\phi_{N}+\phi_{\Delta}} .
\end{aligned}
$$

The relative fractions of $i$-th baryons, $w_{i}$, depend on temperature only and coincide with corresponding values for the ideal gas of $N+\Delta$ baryons [23].

Using Eq. (46) one obtains the formula for the free energy density

$$
f=\mu_{B} n_{B}-P=n_{B} T\left\{\ln \frac{n_{B}}{\phi_{N}+\phi_{\Delta}}-1+\int_{0}^{n_{B}} \frac{d n_{1}}{n_{1}}\left[Z\left(n_{1}\right)-1\right]\right\} .
$$

This leads to the following equations for the energy density and isochoric heat capacity of the $N+\Delta$ mixture:

$$
\begin{aligned}
& \varepsilon=f-T\left(\frac{\partial f}{\partial T}\right)_{n_{B}}=n_{B} T\langle\xi-1\rangle \\
& C_{V}=\left(\frac{\partial \varepsilon}{\partial T}\right)_{n_{B}}=n_{B}\left[\left\langle x^{2}+3 \xi\right\rangle-\langle\xi-1\rangle^{2}\right] \equiv n_{B} \widetilde{C}(T) .
\end{aligned}
$$

Angular brackets in Eqs. (49) -(50) denote averaging over the concentrations of $N$ and $\Delta$ particles. Namely, we define $\langle A\rangle=\sum_{i=N, \Delta} A_{i} w_{i}$ where $w_{i}$ is introduced in (47) and $A_{i}$ is any quantity characterizing the $i$-th component. In particular, $<\xi>=\xi_{N} w_{N}+\xi_{\Delta} w_{\Delta}$ where $\xi_{i}$ is defined in Eq. (12). As one can see from (49) -(50), the energy and heat capacity densities are the same as in the ideal gas of $N+\Delta$ particles [23]. 


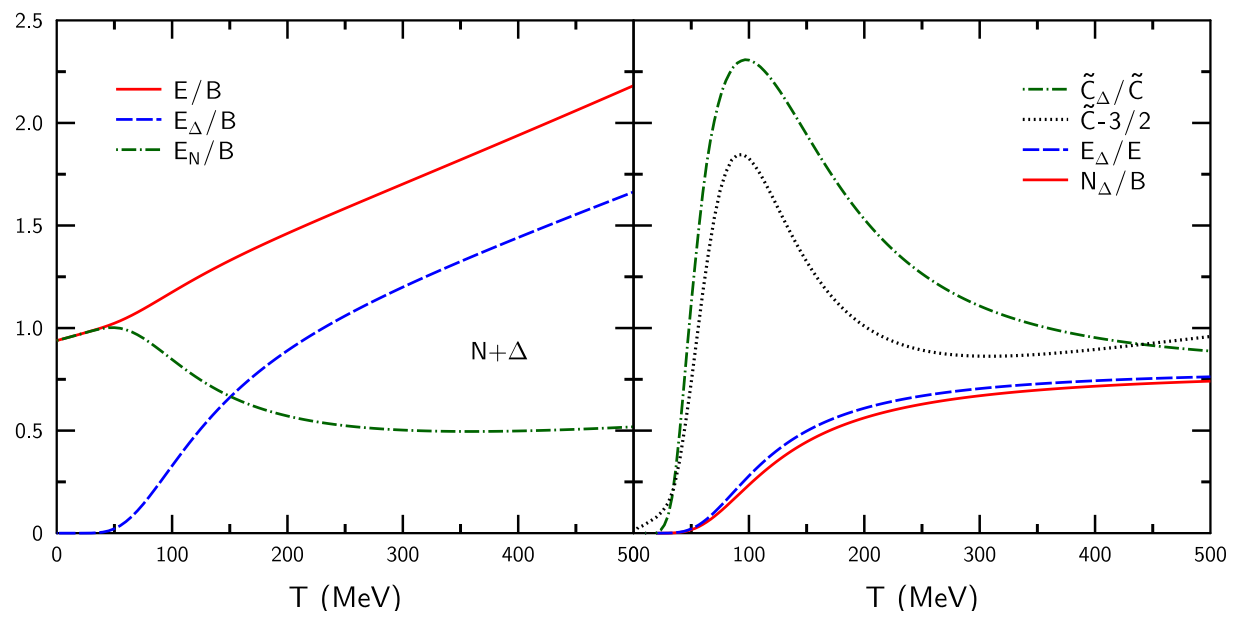

FIG. 7: (Color online) Left panel: the average energy per baryon of $N+\Delta$ matter $E / B$ and partial contributions from nucleons and $\Delta$ 's as functions of temperature (all energies are given in $\mathrm{GeV}$ ). Right panel: the dashed, solid and dash-dotted curves show, respectively, relative contributions of resonances to energy, baryon charge and heat capacity. The dotted line shows temperature dependence of the total heat capacity per baryon minus $3 / 2$.

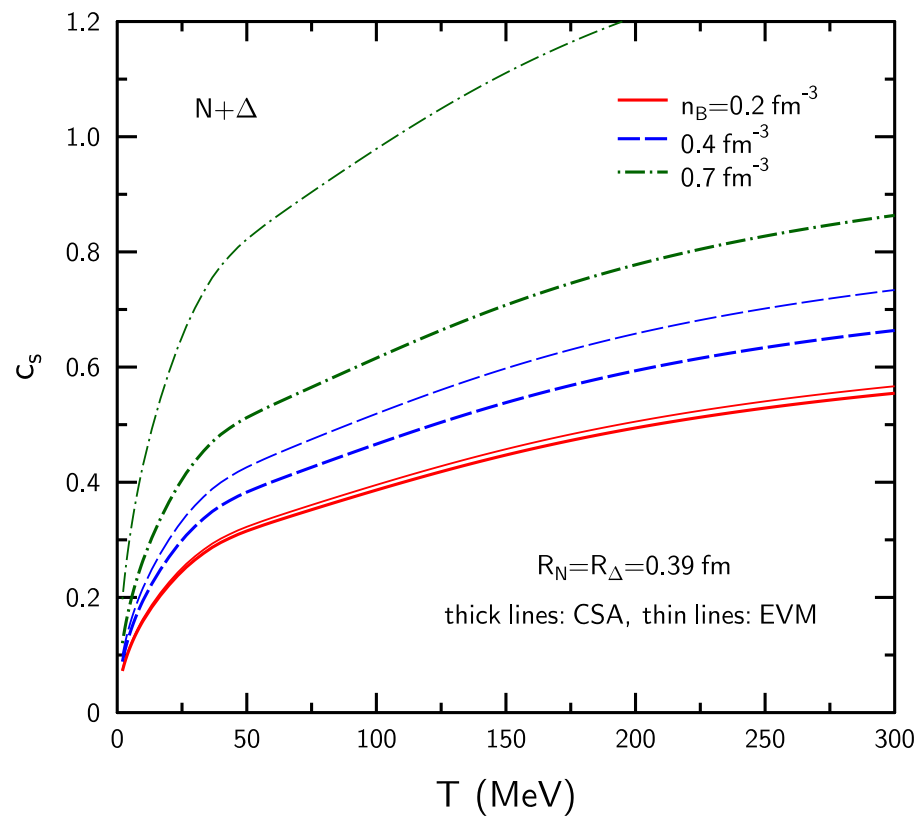

FIG. 8: (Color online) Sound velocity of $N+\Delta$ matter as a function of temperature for several values of baryon density $n_{B}$. Thick and thin curves give the results of CSA and EVM, respectively.

The left panel of Fig. 7 shows the temperature dependence of the total energy per 
baryon $E / B$ as well as the partial contributions to this quantity, $E_{i} / B=T\left(\xi_{i}-1\right) w_{i}$ $(i=N, \Delta)$. At fixed baryon charge $B=N_{N}+N_{\Delta}$ the equilibrium number of $\Delta$ 's increases with temperature (see the right panel). According to Fig. [7, excitation of resonances becomes important at $T \gtrsim 50 \mathrm{MeV}$. It is interesting to note that the nucleon part of energy, $E_{N}$, drops with temperature ${ }^{7}$ in the interval of $T$ approximately between 50 and $350 \mathrm{MeV}$. Introducing the partial components $\widetilde{C}_{i}=B^{-1} d E_{i} / d T$ of the total heat capacity $\widetilde{C}$ we conclude that the nucleon contribution, $\widetilde{C}_{N}=\widetilde{C}-\widetilde{C}_{\Delta}$, is negative in the above mentioned interval of $T$. One can see from Fig. 7 that $\widetilde{C}_{\Delta}>\widetilde{C}$ in this region.

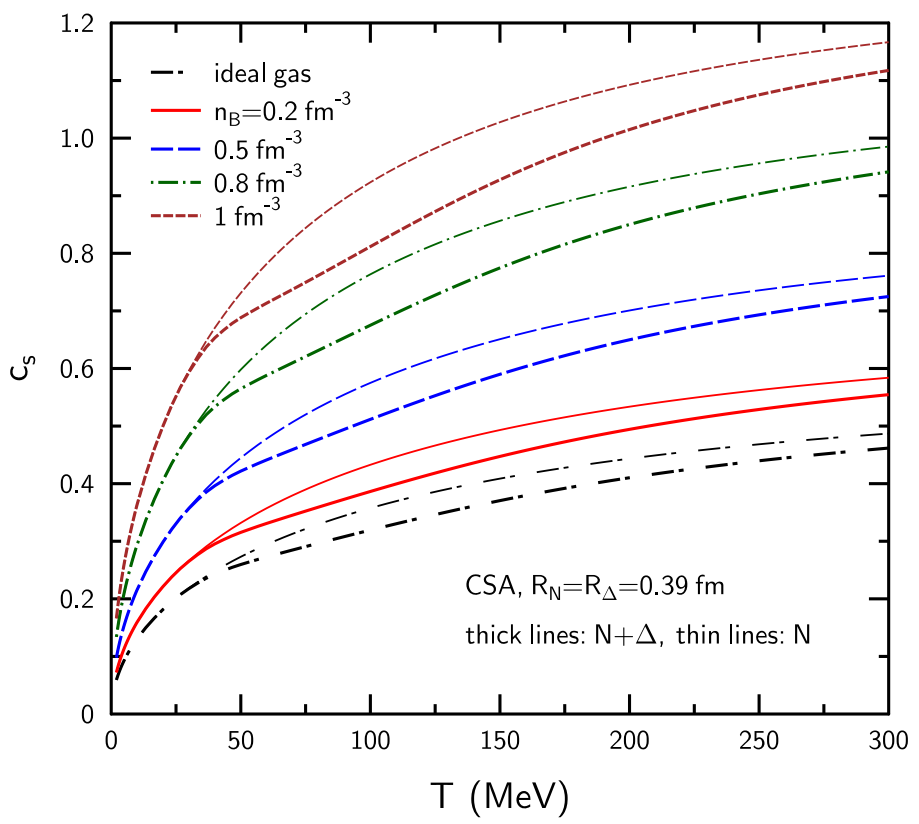

FIG. 9: (Color online) Sound velocities of baryon matter as functions of temperature for different values of baryon density $n_{B}$. Thin and thick lines correspond, respectively, to the nucleon matter and to the $N+\Delta$ mixture. All calculations are made in CSA.

Now we calculate the sound velocity of equilibrium hadronic matter by using the general formula

$$
c_{s}^{2}=\frac{1}{\varepsilon+P}\left[n_{B}\left(\frac{\partial P}{\partial n_{B}}\right)_{T}+\frac{T}{C_{V}}\left(\frac{\partial P}{\partial T}\right)_{n_{B}}^{2}\right] .
$$

This expression is derived in the Appendix using Eq. (14) and basic thermodynamic iden-

7 This occurs because the growth of the nucleon single particle energy, $E_{N} / N_{N}=T\left(\xi_{N}-1\right)$, with raising $T$ is compensated by a stronger decrease of the number of nucleons $N_{N}(T)=w_{N}(T) B$. 
tities $^{8}$. Note that calculating $c_{s}$ from Eq. (51) does not require an explicit form of the Poisson adiabat: one should know only $P$ and $\varepsilon$ as well as their partial derivatives with respect to $T$ and $n_{B}$. We would like to stress that Eq. (51) is applicable for any form of short-range interaction, for any number of hadronic species (including antibaryons and strange particles) and can be used even in the case of quantum statistics.

For a given EoS, one can use Eq. (51) to check constraints imposed by the causality condition $c_{s} \leqslant 1$. For example, for the polytropic EoS $P=\alpha n_{B}^{\gamma}$ at zero temperature, Eq. (51) predicts that $c_{s}^{2}=P^{\prime}\left(n_{B}\right)\left[\int_{0}^{n_{B}} \frac{d n}{n} P^{\prime}(n)\right]^{-1}=\gamma-1$. Therefore, in this case the parameter $\gamma$ should satisfy the condition ${ }^{9} 1 \leqslant \gamma \leqslant 2$.

One can calculate the sound velocity of the $N+\Delta$ mixture using (51) and formulae for $P, \varepsilon, C_{V}$ derived in this section. We arrive at the following result

$$
c_{s}^{2}=\frac{\left(n_{B} Z\right)^{\prime}+Z^{2} \widetilde{C}^{-1}}{<\xi>+Z-1},
$$

where prime denotes the derivative with respect to $n_{B}$. Note that this formula can also be obtained from Eq. (31) if one replaces $n, \xi$ by $n_{B},<\xi>$ and uses, instead of (13), the expression (50) for $\widetilde{C}$.

Figure 8 shows the results of $c_{s}$-calculation in the EVM and CSA. We choose the hadron hard-core radius $R=0.39 \mathrm{fm}$. Again one can see that the CSA predicts smaller sound velocities than the EVM. As compared to the EVM, the sound velocity in the CSA increases with $n_{B}$ much slower, but the temperature dependence is rather similar. In Fig. 9 we compare the sound velocities of the nucleonic and $N+\Delta$ matter. Both calculations are made in the CSA. The ideal gas results are obtained by taking the limit $n_{B} \rightarrow 0$. One can see that inclusion of resonances leads to a noticeable reduction of sound velocities at $T \gtrsim 50 \mathrm{MeV}$. For realistic temperatures $T \lesssim 200 \mathrm{MeV}$, superluminal values $c_{s}>1$ appear only at baryon densities $n_{B} \gtrsim 1 \mathrm{fm}^{-3}$.

The $N+\Delta$ mixture considered so far can not be regarded as a realistic system at high temperatures. In this case mesons will be copiously produced due to inelastic collisions of baryons and decays of resonances. To take these processes into account, below we study the EoS of a three-component $\pi+N+\Delta$ mixture. In this study we again assume that

\footnotetext{
8 A non-relativistic version of (51) has been suggested in Ref. [20].

9 The causal limit for the polytropic EoS was first considered in Ref. [21].
} 
hadrons interact via HSI and neglect possible differences in baryonic radii, i.e. we take $R_{N}=R_{\Delta}=R$. To estimate the sensitivity to the pion size, we consider two limiting cases. First, we assume equal radii for all species, i.e. choose $R_{\pi}=R$, and then we investigate the $\pi+N+\Delta$ mixture with point-like pions $\left(R_{\pi}=0\right)$.

\section{The $\pi+N+\Delta$ matter (same sizes of hadrons)}

In this section we consider the $\pi+N+\Delta$ mixture assuming equal sizes of all hadrons. In this case one can find shifts of thermodynamic functions in the same way as in Sec. IIC for a one-component system. The only difference is that instead of particle density $n$ one should substitute the total density of hadrons $n_{\pi}+n_{B}$. As a result, we obtain the relations

$$
\begin{aligned}
& P=n T Z(n), \quad n=n_{\pi}+n_{B}, \\
& \mu_{i}=T\left[\ln \frac{n_{i}}{\phi_{i}(T)}+\psi(n)\right],
\end{aligned}
$$

for pressure and chemical potentials of particle species $i=\pi, N, \Delta$. Using further Eq. (11), one gets the equations for equilibrium pion density

$$
n_{\pi}=\phi_{\pi}(T) e^{-\psi\left(n_{\pi}+n_{B}\right)}
$$

and for baryon chemical potential

$$
\mu_{B}=T\left[\ln \frac{n_{B}}{\phi_{N}+\phi_{\Delta}}+\psi\left(n_{\pi}+n_{B}\right)\right] .
$$

Solving (55) with respect to $n_{\pi}$ and substituting the result into (53) gives the equilibrium pressure $P=P\left(T, n_{B}\right)$ of the considered mixture. Similarly to Sec. IIIB, one can show that equilibrium fractions $n_{N} / n_{B}$ and $n_{\Delta} / n_{B}$ are the same as in the ideal $N+\Delta$ gas (see Eq. (47)) . According to Eq. (55), interaction with baryons leads to suppression of pion density as compared to pure pion gas $\left(n_{B}=0\right)$. This is demonstrated in Fig. 10 (see the solid and short-dashed curves).

Calculating further the free energy density $f=\mu_{B} n_{B}-P$ and using Eqs. (6) , (55) -(56) lead to the following equations for the energy density and heat capacity

$$
\begin{aligned}
& \varepsilon=T\left[n_{B}\langle\xi-1\rangle+n_{\pi}\left(\xi_{\pi}-1\right)\right], \\
& C_{V}=n_{B}\left[\left\langle x^{2}+3 \xi\right\rangle-\langle\xi-1\rangle^{2}\right]+n_{\pi}\left[x_{\pi}^{2}+3 \xi_{\pi}-\left(\xi_{\pi}-1\right)^{2}(1-\chi)\right],
\end{aligned}
$$




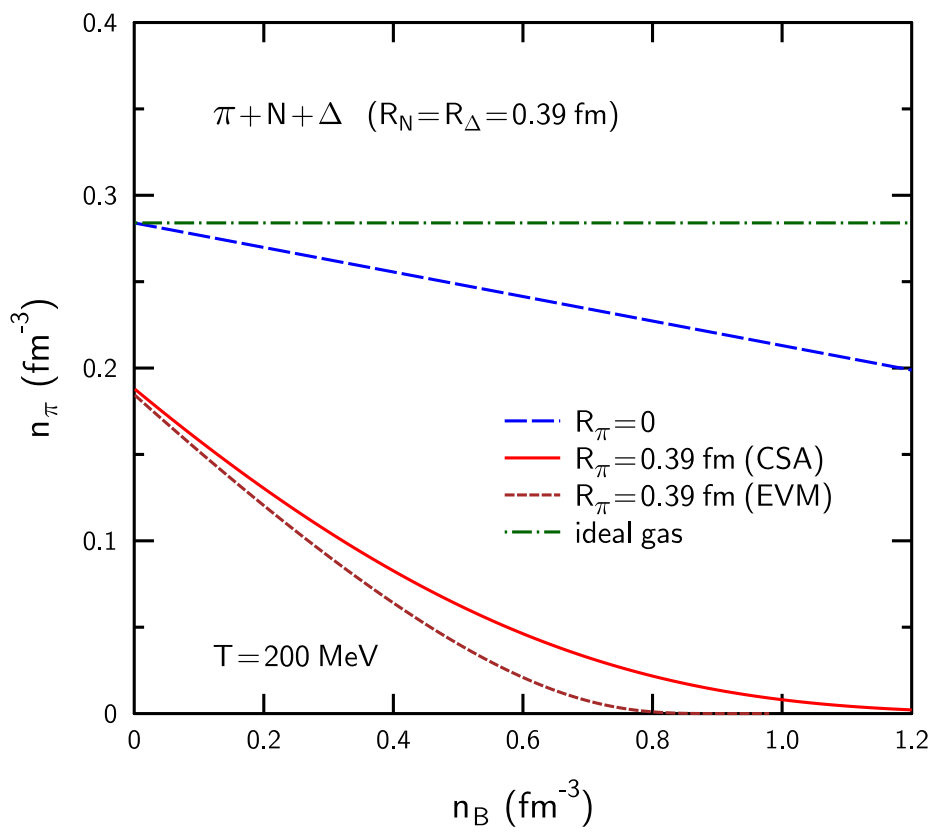

FIG. 10: (Color online) Equilibrium pion density in $\pi+N+\Delta$ mixture as a function of baryon density for $T=200 \mathrm{MeV}$. The solid and short-dashed curves are calculated within CSA and EVM assuming equal sizes of hadrons. The long-dashed line is obtained in the limit of point-like pions. The dashed-dotted curve corresponds to the ideal gas.

where $\chi=1+\left(\frac{\partial n_{\pi}}{\partial n_{B}}\right)_{T}=\left[1+n_{\pi} \psi^{\prime}(n)\right]^{-1}$ (here and below prime denotes the derivative with respect to $n$ ). Note that Eq. (57) formally corresponds to the ideal $\pi+N+\Delta$ gas, but with reduced pion density $n_{\pi}<n_{\pi}^{\mathrm{id}}=\phi_{\pi}(T)$.

Using Eq. (51), one can calculate the sound velocity of the considered matter. We use the following expressions for the derivatives of pressure

$$
\left(\frac{\partial P}{\partial n_{B}}\right)_{T}=T \chi(n Z)^{\prime}, \quad\left(\frac{\partial P}{\partial T}\right)_{n_{B}}=n Z+\chi(n Z)^{\prime} n_{\pi}\left(\xi_{\pi}-1\right) .
$$

The results of $c_{s}$-calculation are shown by the solid lines in Fig. 11 for two values of temperature. The local minima of $c_{s}$ at $n_{B} \sim 0.2 \mathrm{fm}^{-3}$ appear due to a non-monotonic behavior of the total density $n_{\pi}+n_{B}$ as a function of $n_{B}$. Again one can see that compared to EVM, the region of superluminal sound velocities in CSA is shifted to higher baryon densities. 


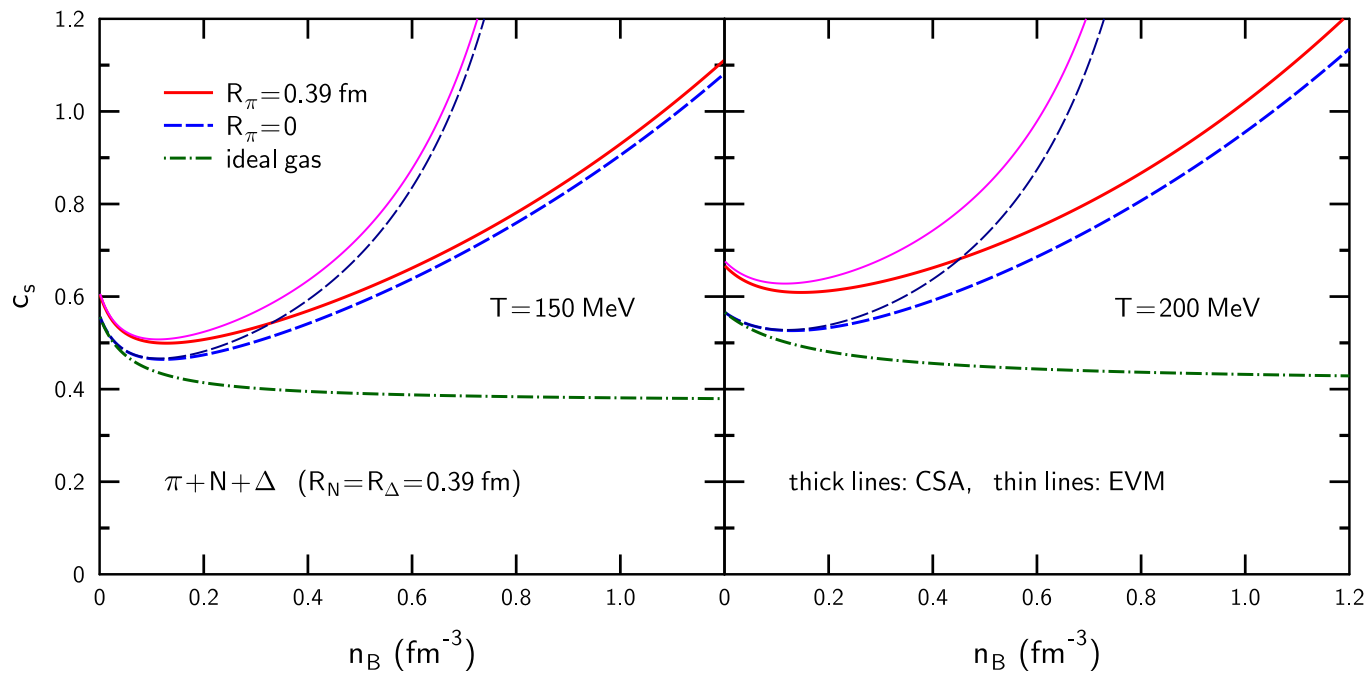

FIG. 11: (Color online) Sound velocity of $\pi+N+\Delta$ matter as a function of baryon density for $T=150 \mathrm{MeV}$ (left panel) and $200 \mathrm{MeV}$ (right panel). Thick and thin curves give, respectively, the results of CSA and EVM. The solid lines correspond to the case of equal sizes of baryons and pions. The dashed lines show the results in the limit of point-like pions. The dashed-dotted curves correspond to the ideal gas.

\section{The $\pi+N+\Delta$ mixture with point-like pions}

Finally we consider the limiting case of point-like pions $\left(R_{\pi}=0\right)$. In accordance with Eq. (44), in this case one can represent pressure of the $\pi+N+\Delta$ system as

$$
P=P\left(T, n_{\pi}, n_{N}, n_{\Delta}\right)=T\left[\frac{n_{\pi}}{1-\eta}+n_{B} Z\left(n_{B}\right)\right]
$$

where $\eta=v n_{B}\left(v\right.$ is the proper volume of a baryon) and $n_{B}=n_{N}+n_{\Delta}$. The compressibility factor $Z$ describes the contribution of baryon interactions. Below we use the parametrizations of $Z$ from Eqs. (44), (5). Substituting $\Delta P=P-\left(n_{\pi}+n_{B}\right) T$ into Eq. (43), one can write the shift of free energy density as follows

$$
\Delta f=f-T \sum_{i=\pi, N, \Delta} n_{i}\left[\ln \frac{n_{i}}{\phi_{i}(T)}-1\right]=T\left\{n_{\pi} \ln (1-\eta)^{-1}+n_{B} \int_{0}^{n_{B}} \frac{d n_{1}}{n_{1}}\left[Z\left(n_{1}\right)-1\right]\right\} .
$$

Using further the conditions of chemical equilibrium

$$
\mu_{\pi}=\frac{\partial f}{\partial n_{\pi}}=0, \quad \mu_{B}=\frac{\partial f}{\partial n_{N}}=\frac{\partial f}{\partial n_{\Delta}},
$$




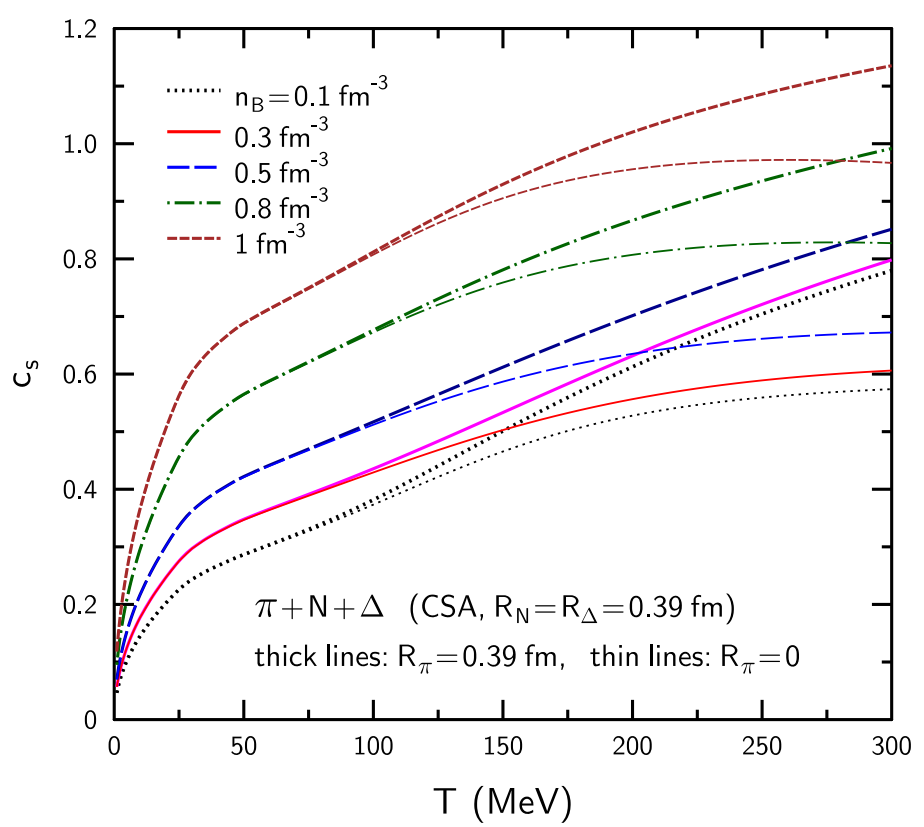

FIG. 12: (Color online) Sound velocity of $\pi+N+\Delta$ matter as a function of temperature for different values of baryon density $n_{B}$. Thick and thin lines are calculated within the CSA assuming, respectively, the pion radii $R_{\pi}=0.39 \mathrm{fm}$ and $R_{\pi}=0$.

one gets the equations for equilibrium densities $n_{i}(i=\pi, N, \Delta)$ as functions of $T$ and $n_{B}$. In this way we obtain the same formulae for $n_{N}$ and $n_{\Delta}$ as for $N+\Delta$ matter (see Eq. (47)).

Inclusion of pions modifies the baryon chemical potential as compared to the $N+\Delta$ system. It is is given by Eq. (46) with the additional term, $\delta \mu_{B}=T \phi_{\pi} v$, in the r.h.s. This contribution has a clear physical meaning. Indeed, to add one baryon to the system of point-like pions, one should create a cavity of volume $v$. At fixed temperature this requires the additional energy (work) $\delta E=P_{\pi} v$, where $P_{\pi}=T \phi_{\pi}$ is the partial pressure of pions (see below). Therefore, the baryon chemical potential should be shifted by the value $\delta \mu_{B}=P_{\pi} v$. Note that this shift may be significant even at small $n_{B}$.

Equilibrium values of pion density and pressure can be written as

$$
n_{\pi}=\phi_{\pi}(T)(1-\eta), \quad P=T\left[\phi_{\pi}(T)+n_{B} Z\left(n_{B}\right)\right]
$$

The last factor in the first equality describes the reduction of volume, available to pions. A linear decrease of $n_{\pi}$ as a function of $n_{B}$ is clearly seen in Fig. 10. One can also obtain the explicit formulae for $\varepsilon$ and $C_{V}$. They are given by Eqs. (57)-(58) after substituting $\chi=1$. 
Using further Eq. (51) we get the equation for sound velocity squared

$$
c_{s}^{2}=\frac{n_{B}\left(n_{B} Z\right)^{\prime}+C_{V}^{-1}\left(\xi_{\pi} \phi_{\pi}+n_{B} Z\right)^{2}}{n_{B}[<\xi>+Z-1]+\phi_{\pi}\left[\xi_{\pi}(1-\eta)+\eta\right]} .
$$

At small $T$, when $\phi_{\pi} \ll 1$ one obtains Eq. (52) for the sound velocity of the $N+\Delta$ mixture. In Figs. 11,12 we compare the results of $c_{s}$-calculations for $R_{\pi}=0.39 \mathrm{fm}$ and $R_{\pi}=0$. As expected, at fixed $T$ and $n_{B}$ the sound velocity increases with $R_{\pi}$. A realistic value for $R_{\pi}$ is somewhere between the two considered cases. Based on the results presented in Fig. 12 we conclude that the EoS for the $\pi+N+\Delta$ mixture remains causal up to the baryonic densities where the deconfinement phase transition is expected.

\section{CONCLUSIONS AND OUTLOOK}

In this paper we have investigated the EoS and sound velocities of one- and multicomponent hadronic systems with HSI. It is shown that widely used excluded volume models become unrealistic at packing fractions exceeding about 0.2 . We demonstrate that the Carnahan-Starling EoS is much softer and can be applied at much higher densities. Moreover, the sound velocity calculated for this EoS shows the acausal behaviour only at very high baryon densities, presumably in the region of quark-gluon phase transition. Comparing the sound velocities in hot and dense hadronic systems with different compositions of particles, we have studied the sensitivity of the EoS of strongly interacting matter to the formation of pions and baryon resonances.

In the future we are going to perform similar analysis for more realistic systems which include heavier mesons, baryons and antibaryons. Using the Carnahan-Starling EoS and the approach suggested in Ref. [6] we plan to investigate the sensitivity of phase diagram of strongly interacting matter to finite sizes of hadrons. In this way one can construct a realistic EoS suitable for hydrodynamical modeling of heavy-ion collisions. In particular, one may

perform simulations similar to Ref. [24] to analyze possible signatures of the deconfinement phase transition.

It would be interesting to extend this analysis beyond the limits of Boltzmann approximation and include quantum-statistical effects for hadronic systems with HSI. These effects should be certainly important for dense mater at low temperatures (e.g. in compact stars). Some attempts in this direction have been made [6, 8, 25, 26] within the excluded 
volume approach.

\section{APPENDIX A. General formula for sound velocity}

Let us assume that one knows pressure $P$ and energy density $\varepsilon$ of an equilibrated matter as functions of temperature $T$ and the baryon density $n_{B}$. In this case one can easily calculate the adiabatic sound velocity $c_{s}$.

From the thermodynamic relation [14] $d \varepsilon=(\varepsilon+P) d n_{B} / n_{B}+n_{B} T d \sigma$ one gets the expressions

$$
\left(\frac{\partial \varepsilon}{\partial n_{B}}\right)_{\sigma}=\frac{\varepsilon+P}{n_{B}}, \quad\left(\frac{\partial \sigma}{\partial T}\right)_{n_{B}}=\frac{C_{V}}{n_{B} T},
$$

where $C_{V}=(\partial \varepsilon / \partial T)_{n_{B}}$ is the isochoric heat capacity.

Using the identity $d P / n_{B}=\sigma d T+d \mu_{B}$ one arrives at the relation

$$
\left(\frac{\partial \sigma}{\partial n_{B}}\right)_{T}=\frac{\partial}{\partial n_{B}}\left(\frac{1}{n_{B}} \frac{\partial P}{\partial T}-\frac{\partial \mu_{B}}{\partial T}\right)=-\frac{1}{n_{B}^{2}}\left(\frac{\partial P}{\partial T}\right)_{n_{B}} .
$$

From Eqs. A.1 - A.2) one can write the equation for the temperature derivative in the adiabatic process

$$
\left(\frac{\partial T}{\partial n_{B}}\right)_{\sigma}=-\left(\frac{\partial \sigma}{\partial n_{B}}\right)_{T}\left(\frac{\partial \sigma}{\partial T}\right)_{n_{B}}^{-1}=\frac{T}{n_{B} C_{V}}\left(\frac{\partial P}{\partial T}\right)_{n_{B}}
$$

Note that this equation takes the form (30) for classical nucleons with HSI.

Equation (14) can be represented as follows

$$
c_{s}^{2}=\left(\frac{\partial P}{\partial n_{B}}\right)_{\sigma}\left(\frac{\partial \varepsilon}{\partial n_{B}}\right)_{\sigma}^{-1}=\frac{n_{B}}{\varepsilon+P}\left[\left(\frac{\partial P}{\partial n_{B}}\right)_{T}+\left(\frac{\partial P}{\partial T}\right)_{n_{B}}\left(\frac{\partial T}{\partial n_{B}}\right)_{\sigma}\right] .
$$

Substituting (A.3) into (A.4) gives the formula (51) of the main text.

\section{Acknowledgments}

The authors thank M.I. Gorenstein, A.I. Ivanytskyi and V.V. Sagun for useful discussions. K.A.B. and I.N.M. acknowledge a financial support provided by the Helmholtz International Center for FAIR (Germany). A partial support from the grant NSH-932.2014.2 is acknowledged by I.N.M. and L.M.S. K.A.B. acknowledges a partial support of the program 
"On perspective fundamental research in high-energy and nuclear physics" launched by the Section of Nuclear Physics of NAS of Ukraine.

[1] J.D. van der Waals, in Nobel lectures in physics 1901-1921, Elsevier, Amsterdam, 1967.

[2] J.P. Hansen and I.R. McDonald, Theory of simple fluids, Academic Press, Amsterdam, 2006.

[3] A. Mulero (ed.), Theory and simulation of hard sphere fluids and related systems, Lect. Notes Phys. 753, Springer-Verlag, Berlin, 2008.

[4] J. Cleymans, K. Redlich, H. Satz, and E. Suhonen, Z. Phys. C 33, 151 (1983).

[5] D.H. Rischke, M.I. Gorenstein, H. Stöcker, and W. Greiner, Z. Phys. C 51, 485 (1991).

[6] L.M. Satarov, M.N. Dmitriev, and I.N. Mishustin, Yad. Fiz. 72, 1444 (2009) [Phys. Atom. Nucl. 72, 1390 (2009)].

[7] K.A. Bugaev, Nucl. Phys. A 804, 251 (2008).

[8] J. Steinheimer, S. Schramm, H. Stöcker, J. Phys. G 38, 035001 (2011).

[9] K.A. Bugaev, D.R. Oliinychenko, J. Cleymans, A.I. Ivanytskyi, I.N. Mishustin, E.G. Nikonov, and V.V. Sagun, Eur. Phys. Lett. 104, 22002 (2013).

[10] D.R. Oliinychenko, K.A. Bugaev, and A.S. Sorin, Ukr. J. Phys. 58, 211 (2013).

[11] S. Kagiyama, A. Minaka, A. Nakamura, Prog. Theor. Phys. 89, 1227 (1993).

[12] C.P. Singh, B.K. Patra, K.K. Singh, Phys. Lett. B 387, 680 (1996).

[13] M.I. Gorenstein, Phys. Rev. C 86, 044907 (2012).

[14] L.D. Landau and E.M. Lifshitz, Statistical physics, Pergamon Press, Oxford, 1980.

[15] N.F. Carnahan and K.E. Starling, J. Chem. Phys. 51, 635 (1969).

[16] L.D. Landau and E.M. Lifshitz, Fluid mechanics, Pergamon Press, Oxford, 1987.

[17] A. Mulero, C.A. Faúndez, and F. Cuadros, Mol. Phys. 97, 453 (1999).

[18] Y. Aoki et al., JHEP 0906, 088 (2009).

[19] A. Bazavov et al. (HotQCD Collaboration), Phys. Rev. D 85, 054503 (2012).

[20] Y. Rosenfeld, J. Phys.: Condens. Matter 11, L71 (1999).

[21] Ya.B. Zeldovich, Sov. Phys. JETP 14, 1143 (1962).

[22] B.J. Alder, J. Chem. Phys. 23, 263 (1955).

[23] V.M. Galitsky and I.N. Mishustin, Yad. Fiz. 29, 363 (1979).

[24] A.V. Merdeev, L.M. Satarov, and I.N. Merdeev, Phys. Rev. C 84, 014907 (2011). 
[25] G.D. Yen, M.I. Gorenstein, W. Greiner, and S.N. Yang, Phys. Rev. C 56, 2210 (1997).

[26] S. Wheaton, J. Cleymans, and M. Hauer, Comp. Phys. Comm. 180, 84 (2009). 